\title{
Endocrine manifestations of malignancy
}

\author{
Nadia Yousaf \\ Sanjay Popat
}

Nadia Yousaf MB BS MRCP MD is a Consultant Medical Oncologist at the Royal Marsden Hospital, London, UK. Competing interests: NY has received travel expenses from Pfizer and Chugai, and lecture fees from Astra Zeneca.

Sanjay Popat BSc MB BS FRCP PhD is a Consultant Medical Oncologist at the Royal Marsden Hospital, London, UK and Reader at Imperial College London, UK. Competing interests: SP is consultant to Ariad, AstraZeneca, Boehringer Ingelheim, BMS, Eli Lilly, MSD, Roche, Pfizer, Novartis.

\begin{abstract}
Endocrine manifestations of cancer are usually paraneoplastic syndromes (i.e. rare systemic manifestations of malignancy caused by non-mass cancer effects). The pathobiology involves the production of hormone and related substances that act in an endocrine or paracrine manner, resulting in systemic manifestations. Two major mechanisms are observed: ectopic hormone production by nonendocrine organs and direct hormone secretion from endocrine malignancies. The most common paraneoplastic syndromes include hypercalcaemia, Cushing's syndrome and syndrome of inappropriate antidiuretic hormone secretion. These may be the presenting feature of an underlying malignancy. Rarer syndromes result from ectopic production of less frequently observed bioactive proteins (e.g. growth hormone-releasing hormone, human chorionic gonadotrophin, insulin-like growth factors, renin, vasoactive intestinal peptide) and iatrogenic causes. Here, we review the spectrum of common and rare endocrine manifestations of malignancy.
\end{abstract}

Keywords

Cushing's syndrome; hypercalcaemia; paraneoplastic syndrome; syndrome of inappropriate antidiuretic hormone

\section{Key points}

- Hypercalcaemia and syndrome of inappropriate antidiuretic hormone secretion are the most common paraneoplastic endocrine manifestations of cancer

- Appropriate investigations should be conducted to exclude other differential diagnoses

- Endocrine manifestations most frequently occur in cancers with neuroendocrine differentiation

- Endocrine abnormalities can also be associated with drugs used to treat cancer

\section{Background}

Primary endocrine tumours are uncommon, but many malignancies have systemic, endocrine effects, termed paraneoplastic syndromes, defined as symptoms resulting from substances released by cancer cells that disrupt normal cell function. Ectopic production of bioactive substances (usually hormone precursors or analogues) is the most common cause and is mainly seen in tumours with neuroendocrine differentiation. Such cells are distributed throughout the body (e.g. lung, gastrointestinal tract, thyroid, adrenals, breast, prostate, skin) and physiologically produce biogenic peptides. Associated malignancies (e.g. carcinoids, small cell lung cancer (SCLC)) account for most ectopic hormone-producing tumours.

Early recognition of paraneoplastic syndrome is vital for the timely diagnosis of underlying malignancy. Florid, untreated paraneoplastic syndromes can cause significant morbidity and mortality, and delay definitive cancer treatment.

\section{Common endocrine-related manifestations of cancer Hypercalcaemia of malignancy}

Paraneoplastic hypercalcaemia is common, affecting about $20-30 \%$ of individuals with malignancy. One of the following mechanisms will be responsible: ${ }^{1}$ 
1. Ectopic production of parathyroid hormone-related peptide (PTHrP), resulting in increased osteoblastic activity, bone resorption, renal tubular calcium resorption and phosphate excretion. This is most commonly associated with haematological malignancies as well as some solid organ carcinomas: squamous cell (lung, head, neck), renal, bladder and breast.

Diagnosis is based on high PTHrP, low parathyroid hormone (PTH), low phosphate and low/normal 1,25-dihydroxyvitamin $\mathrm{D}_{3}$.

2. Increased 1a-hydroxylase activity in some lymphomas causes unregulated extra-renal production of 1,25-dihydroxyvitamin $\mathrm{D}_{3}$.

Diagnosis is based on normal/raised serum phosphate, low PTH and high 1,25-dihydroxyvitamin $\mathrm{D}_{3}$.

3. Osteolytic bone metastases result in localized production of cytokines, leading to bone resorption. Most commonly associated with breast cancer and myeloma.

Diagnosis is based on low PTH/PTHrP and low/normal 1,25-dihydroxyvitamin $\mathrm{D}_{3}$

4. Ectopic PTH secretion in ovarian, lung and papillary thyroid pancreatic carcinomas (very rare).

Clinical features - these include anorexia, nausea, vomiting, constipation, abdominal pain, polyuria, polydipsia, confusion and coma.

Investigations and management of malignant hypercalcaemia - patients with mild hypercalcaemia (adjusted calcium $<3 \mathrm{mmol} / \mathrm{litre}$ ) should be hydrated, and drugs such as thiazide diuretics and lithium should be reviewed.

Patients with moderate hypercalcaemia (adjusted calcium 3-3.5 mmol/litre) should be treated with intravenous saline hydration and consideration given to administering a bisphosphonate such as zolendronic acid (onset of action 2-4 days) or a receptor activator of nuclear factor kappa-B (RANK) ligand antibody such as denosumab (if creatinine clearance is $<30 \mathrm{ml} / \mathrm{minute}$ ).

Patients with severe hypercalcaemia (adjusted calcium $>3.5 \mathrm{mmol} /$ litre) or moderate to severe symptoms should be treated as above. In addition, calcitonin ( $4 \mathrm{mg} / \mathrm{kg}$ four times a day) can provide short-term symptomatic benefit (onset of action 12-48 hours), although the response decreases with repeated administration.

If there is no response to zolendronic acid after 7 days, it can be repeated. If the hypercalcaemia is bisphosphonate-refractory, consider a RANK ligand antibody. ${ }^{2}$ Hypercalcaemia secondary to lymphoma can be treated with corticosteroids and treatment of the lymphoma.

\section{Ectopic adrenocorticotrophic hormone (ACTH) syndrome}

Cushing's syndrome ${ }^{3}$ is cause by ectopic ACTH in tumours with neuroendocrine differentiation, most commonly SCLC and pulmonary carcinoids.

Clinical features - these include hyperglycaemia, hypokalaemia, hypertension, proximal myopathy, centropedal adiposity and ecchymoses.

performed:

Investigations - if Cushing's syndrome is suspected, at least one of the following tests should be

-late night salivary cortisol (two separate occasions)

-24-hour free urinary cortisol (two separate occasions)

- overnight $1 \mathrm{mg}$ dexamethasone suppression test or 48-hour $2 \mathrm{mg}$ dexamethasone suppression test. Cushing's syndrome is unlikely if these tests are negative. If results are positive, measure serum ACTH. Management of Cushing's syndrome - for localized disease, consider surgical resection. For patients with non-resectable disease, cortisol synthesis can be suppressed with using metyrapone or ketoconazole.'

\section{Syndrome of inappropriate antidiuretic hormone (SIADH) secretion}

In malignancy-related SIADH, ${ }^{4}$ the most common mechanism is ectopic antidiuretic hormone (ADH) production. SIADH is most commonly associated with SCLC. Other less common malignancies associated with SIADH include non-SCLCs, head and neck carcinomas and sarcomas. Cytotoxic drugs such as cyclophosphamide, vinca alkaloids and platinums can enhance pituitary ADH release. 
Clinical features - these are shown in Table 1.

Table 1. Clinical features of hyponatraemia

\begin{tabular}{|l|l|}
\hline Level of hyponatraemia & Signs and Symptoms \\
\hline Mild (Na $130-135 \mathrm{mmol} /$ litre) & Frequently asymptomatic \\
\hline Moderate $(\mathrm{Na} 121-129 \mathrm{mmol} / \mathrm{litre})$ & $\begin{array}{l}\text { Can be asymptomatic or produce fatigue, } \\
\text { nausea, lethargy, confusion, forgetfulness, } \\
\text { dizziness, gait disturbance and muscle } \\
\text { cramps }\end{array}$ \\
\hline Severe $(\mathrm{Na}<121 \mathrm{mmol} /$ litre $)$ & As above plus coma and seizures \\
\hline
\end{tabular}

comprise:

Investigations - the diagnosis of SIADH is supported by Bartter and Schwartz criteria, which

•euvolaemia

-not on diuretics

-normal thyroid and adrenal function

-decreased plasma osmolality $(<280 \mathrm{mOsm} / \mathrm{kg})$

-inappropriately concentrated urine $(>100 \mathrm{mOsm} / \mathrm{kg})$

-elevated urinary sodium (>40 mmol/litre).

Management - the management of hypernatraemia can be stratified by the following criteria:

-Mild to moderate symptoms - management of the underlying malignancy together with fluid restriction (800 ml/day). If unsuccessful, consider demeclocycline (initially 900-1200 mg per 24 hours in divided doses) or vasopressin-2-receptor antagonists (Tolvaptan 15-60 mg once daily)

-In symptomatic chronic hyponatraemia, the serum sodium must be corrected slowly (4-6

$\mathrm{mmol} /$ litre per 24 hours) because of the risk of osmotic demyelination syndrome.

-In patients with severe symptoms, i.e. seizure and coma, $100 \mathrm{ml}$ of $3 \%$ saline can be given as a

bolus over 10 minutes and repeated 15 minutes later if there has been no clinical improvement.

\section{Other rare endocrine-related manifestations of cancer}

Table 2 contains a summary of rare endocrine manifestations of cancer. ${ }^{5}$ In addition, many endocrine toxicities from drugs used in the treatment of cancer have been documented (e.g. Cushing's syndrome from glucocorticoids, hypocalcaemia from platinum-based therapy or steroidal CYP17A1 inhibitors such as Abiraterone, and diabetes insipidus from ifosfamide). More recently, drug-related endocrine toxicities with newer targeted anti-cancer therapies have been observed: for example, crizotinib (an anaplastic lymphoma kinase inhibitor) can cause central hypogonadism in men who require testosterone replacement, and immunotherapy drugs (anti-phosphodiesterase 1 (PD1)/PD-L1 antibodies or anticytotoxic T-lymphocyte associated protein 4 (CTLA4) antibodies) can cause a diverse range of autoimmune endocrine abnormities such as hypo/hyperthyroidism or pan-hypophysitis.

Table 2

Symptoms and management of rare paraneoplastic endocrine manifestations of cancer

\section{Hormone Cancers implicated}

$\mathrm{GHrH}$

hCG
pNET, SCLC, lung carcinoid, lung and gastric adenocarcinoma

Teratoma, choriocarcinoma, hepatocellular carcinoma

\section{Clinical symptoms}

Acromegaly

Menstrual dysfunction, gynaecomastia, precocious puberty, symptoms of

\section{Biochemical abnormalities}

$\uparrow$ Growth hormone

$\uparrow \mathrm{hCG}$

Normal TSH/T3

\section{Management}

Treat underlying malignancy

Treat underlying malignancy

Propranolol for thyrotoxicosis 



\section{KEY REFERENCES}

1. Clines GA. Mechanisms and treatment of hypercalcaemia of malignancy. Curr Opin Endocrinol Diabetes Obes 2011; 18: 339-46.

2. Hu MI, Glezerman IG, Leboulleux S, et al. Denosumab for treatment of hypercalcaemia of malignancy. J Clin Endocrinol Metab 2014; 99: 3144-52.

3.

3. Newell-Price J, Bertanga X, Grossman AB, Nieman L. Cushing's syndrome. Lancet 2006; 367: 1605-17.

4. Ellison MD, Berl T. The syndrome of inappropriate antidiuresis. N Engl J Med 2007; 356: 206472.

4. 5. DeLellis RA, Xia L. Paraneoplastic endocrine syndromes: a review. Endocr Pathol 2003; 14: 303-17. 


\section{TEST YOURSELF}

To test your knowledge based on the article you have just read, please complete the questions below.

The answers can be found at the end of the issue or online here.

\section{Question 1}

A 56-year-old woman presented acutely with vomiting and abdominal pain. She had a history of breast cancer with bone metastasis She had been taking letrozole (an aromatase inhibitor) for her breast cancer for the previous 2 years.

On clinical examination, she was dehydrated and disorientated in time and place. She was admitted and treated with intravenous fluids and a bisphosphonate (zolendronic acid $4 \mathrm{mg}$ ). The next day, she appeared distressed, more confused and drowsy.

\section{Investigations}

-Initial serum corrected calcium $3.7 \mathrm{mmol} /$ litre $(2.20-2.60)$

-Next-day serum corrected calcium $3.8 \mathrm{mmol} /$ litre $(2.20-2.60)$

\section{What is the most appropriate next step?}

A. Treat again with another bisphosphonate, i.e. pamidronate

B. Administer a RANK ligand antibody, i.e. denosumab

C. Continue intravenous fluids and repeat blood tests the next day

D. Organize a CT scan of the brain

E. Consider administering calcitonin

Correct answer: E. The onset of action of bisphosphonates is 2-4 days; therefore repeating a dose of any bisphosphonate (A) before this time would not be useful. Similarly administering denosumab within this time period would not be helpful and the onset of action of denosumab can take up to 7 days and therefore B would not lead to an immediate improvement in symtpoms. Calcitonin has a faster onset of action although the effect is short lived; therefore it may be useful to manage the patient's symptomatic deterioration while waiting for the bisphosphonate to work. The raised calcium is a sufficient explanation for her symptoms (so D is inappropriate). Her condition justifies additional immediate treatment (so $\mathrm{C}$ is incorrect).

\section{Question 2}

A 67-year-old man presented with confusion, lethargy and gait disturbance. He had completed chemotherapy for extensive stage small cell lung cancer 2 months previously.

\section{Investigations}

-Serum sodium $125 \mathrm{mmol} /$ litre (137-144)

-Serum potassium $4.8 \mathrm{mmol} /$ litre (3.5-4.9)

-Plasma osmolality $260 \mathrm{mosmol} / \mathrm{kg}(278-300)$

-Urine $300 \mathrm{mosmol} / \mathrm{kg}(100-1000)$

Random Serum cortisol 500

\section{What is the most appropriate next step?}
A. Hypertonic saline
B. hydrocortisone IV $100 \mathrm{mg}$
C. Fluid restriction to $800 \mathrm{ml}$ per day
D. Treatment with demeclocycline
E. Vasopressin-2-receptor antagonists

Correct answer: C. The patient has moderate symptoms from syndrome of inappropriate antidiuretic hormone secretion and should therefore be initially managed with strict fluid restriction. If there is no improvement in the sodium after 48 hours, consideration can be given to demeclocycline (D) or, if unsuccessful, a vasopressin-2-receptor antagonist $(E)$. A Is only recommended in an emergency situation 
due to the risk of rapid correction of hyponatraemia and a risk of osmotic demyelination syndrome. B would be appropriate for a diagnosis of addisions disease but the serum cortisol is not supportive of this.

\section{Question 3}

A 25-year-old man presented with gynaecomastia. A chest X-ray showed a mediastinal mass.

\section{Which bedside test might be diagnostically useful?}
A. Blood pressure
B. Spirometry
C. Pregnancy test
D. Pulse oximetry
E. Urinalysis

Correct answer: C. Gynaecomastia and a mediastinal mass may be indicative of a mediastinal teratoma. A pregnancy test can detect the presence of $\beta$-human chorionic gonadotrophin subunits and can therefore be positive in patients with a teratoma.
A Inspection for acanthosis nigricans
B Blood pressure monitoring
C Pregnancy test
D Visual field assessment
E Estimation of testicular size 